\title{
XXXVI. On vaccination. An examination of several of the mis-statements of Dr. Rowley
}

\section{Mr. J.J. Hawkins}

To cite this article: Mr. J. J. Hawkins (1806) XXXVI. On vaccination. An examination of several of the mis-statements of Dr. Rowley, Philosophical Magazine Series 1, 24:95, 204-209, DOI: $10.1080 / 14786440608563365$

To link to this article: http://dx.doi.org/10.1080/14786440608563365

曲 Published online: 18 May 2009.

Submit your article to this journal $\sqsubset \pi$

Џ Article views: 2

Q View related articles $\sqsubset$ 
drogen gas) in the common way, with sulphuric acid, water, and iron filings. This result I experienced several times; the cylinder resting on the copper end of a Galvanic trough consisting of 25 pairs of plates, $2 \frac{1}{4}$ inch. When the nose was placed on a cylinder connected with the zinc end, no such smell was produced. Quere, How was the inflammable air (if any was) produced to make so strong a smell ? The first time I perceived this smell was on Saturday last. $I$ again tried the experiment this evening, and found the same result. I likewise tried it with two troughs, consisting in all of 50 pairs of plates; and then also perceived the smell : but the experiment was from the number of plates so very unpleasant (the shock being great) that I did not continue it very long, and did not determine whether the smell was proportionally strong.

With these 50 pairs of plates another effect occurred, which I had never seen before. I introduced in the circuit a small glass tube filled with milk instead of water, and whilst it remained in its place I did not see any alteration take place; but when I took out the wire, which was a silver one, and had been connected with the zinc end, I found a sort of crust adhering to it which looked like cream. Quere, Was not this curd formed by acid from the zinc end? A gold wire, which was connected with the copper end, I neglected to examine.

April 7, 1806 .

A Friend to Physical Inquiries.

XXXVI. On Vaccination. An Examination of several of the Mis-statements of Dr. Rowley. By Mr. J. J. HawKins, of Istington.

sir,
$H_{\text {Aving for some years seen the most beneficial effects }}$ from the introduction of the cow-pock, as well from the practice of many of my friends, as with several I have myself vaccinated, I felt it a duty, on the first publication of Dr. Rowley's pamphlet, entitled "Cow-Pox Inoculation no Security 
Security against Small-Pox Infection," to examine some of his alleged cases, in order that I might judge for myself, whether I ought to continue favourable, or become adverse to the new inoculation.

It was at first my intention to examine the whole of his cases; but, hearing that the indefatigable Dr. Thornton had undertaken the task, and knowing he was better qualified than myself, I relinquished it, after visiting ten of the 218 cases detailed in Dr. Rowley's publication. Of these ten, there were three grossly misrepresented; four could not be proved to have gone regularly through the cow-pock; of one no information could be had, the father of the child having died a twelvemonth before Dr. Rowley published the case; and two were most pointedly in favour of vaccination.

Dr. Rowley's scandalous pamphlet would have been totally unworthy of the least notice had he not addressed himself to the passions, and thereby wrought upon the credulity of the ignorant and unthinking; which renders it necessary that the cause of truth and humanity should be vindicated.

I understand there are several replies to Dr. Rowley, two only of which have come into my hands; one, a most excellent satirical piece, by "Aculeus;" the other by Dr. Thornton, two reports only of whose labours are as yet published: so far as he has already gone, he has entirely disproved and overturned Dr. Rowley's statements, and exposed the baseness of antivaccinarian proceedings.

From my own observations and practice, I can truly say, I have met with nothing but the most satisfactory evidence. in favour of vaccine inoculation, and therefore I conceive it my duty to promote it by all the means in my power. Although the subject is so well handled by the two authors just mentioned, I think it will be of use to contribute my mite to the general stock of evidence, and shall be glad you will insert in your valuable Magazine the following statc.ment of my examination of the ten cases:

Case 1. The case of Marianne Lewis, which is the 88th in Dr. Rowley's list, and the subject of one of his engravings, I examined with very particular attention.

The doctor states, that she was vaccinated "at the Small- 
Pox Hospital April 1803, and that in June 1804 she broke out in the head, ears, and chest, with cow-pox mange, cow-pux blueish abscesses: when the cold weather came, they were better. In May 1805, the samc cow-pox mange, cow-pox abscesses, blueish in appearance; attacked the child in every part of the body, from the head to the foot; there were nothing but cow-pox mange, cow-pox gatherings of matter, cow-pox ulcers, excoriations : the child was a mere Lazarus, covered all over with sores and disease; a most disgusting spectacle of terrible disease."

On my visiting this child in October 1805, the mother told me that it was vaccinated in May 1803.' There were about 200 vaccinated at the same time. She never carried her to be seen afterwards. There was no regular pustule, but only a little sore without inflammation, which soon healed up. The child was particularly healthy till April 1804, when she har eruptions on the head and breast, which continued four months. She was then quite well, till May last, when the eruptions came again, and extended all over the body : they are now, for the most part, healed. She says also, that Dr. Rowley gave it as his opinion, that the sore was neither cow-puck nor small-pox, but something between both.

From both these statements it appears that the child was inoculated for the cow-pock, and afterwards had an eruption; but the doctor has carefully omitted that, which would have made it no evidence in his favour, namely, that the child did not take the cow-pock, although inoculated for it, and that, for eleven months after the inoculation, she was particularly healthy.

There is some difference also in the time of the eruption: the doctor states it to commence in June, and to become bettor in cold weather; but the mother says it commenced in April, and continued four months, (till August, the hottest montin in the year,) when the child was quite well, till the May following.

From my own examination I am induced to believe that the eruption was very far from being all over the body; but it was sufficient for the doctor's purpose, if he could any way twist facts to farour the antivaccine principles, 
for which end be has most grossly nistrepresented this case.

Case 2. Mr. Joules's son, Dr. Rowley's 36th case, stated as follows:-" Vaccinated at the Small-Pox Hospital; terrible tumour in the face, of which a drawing is given resembling an ox."

I saw this child, with his parents, in November 1805. The father told me that the boy was vaccinated four or five years since: soon afterwards he had a slight breaking out on the face, which continued about a month: from that time he was perfectly well for three or four years, when a swelling came in his cheek, but was attended with so little pain, that it did not hinder his playing in the streets as much as other boys. Mr. Joules said, Dr. Rowley promised to undertake the cure gratis, but never troubled hinself about it after he had the boy's picture. The father and mother both declared they did not think the swelling was caused by the cow-pock. It appeared to me a scrophulous case, and nothing could be further fctched than the notion of its resembling an ox.

Case 3. Mr. Wild's child, the doctor's 135th case, who, he says, took the small-pox, in the natural way, in August 1805, although vaccinated two years lefore.

I saw the mother and child in October 1805. She said the child was vaccinated three or four years before it took the small-pox; but the place of inoculation was very small, and did not appear like the cow-pock she had seen in other persons ; from whence it is evident, although the child was inoculated for the cow-pock, it did not receive it. Is it any wonder then, that the small-pox infection tuok place when the child was exposed to it? Certainly not. And it is therefore a gross misrepresentation of the case, to call it an instance of cow-pock failure.

Case 4. Mr. Colson's grandson, Dr. Rowley's 13\%th case. This he states took the small-pox two years after cow-pox.

In October 1805, I saw Mr. Colson, the grandfather, and also the child's mother, who was but seventeen years of age when her child was vaccinated; and thoughtlessly neglected 
to carry it to the Skinner-street station, where it was inocilated, to be examined so often as required by the rules of the institution. This, she told me, was entered in the books at the station. It therefore cannot be known that the child went regularly through the cow-pock.

Cases 5 and 6. Mrs. Little's two children, Dr. Rowley's 77 th case. He states the small-pox happening three years after vaccination : but from every information I could obtain from the mother and others, there is no proof that they passed regularly through the cow-pock, and consequently they ought not to have been brought forward as cases against vaccination.

Case 7. Mr. Nicholson's boy, the doctor's 35th case: "Small-pox two years after vaccination." The regularity of the vaccination is not proved; and there is no other evidence of the child's having the small-pox, than Dr. Rowley's saying there was one small-pock on its posteriors, from which he could have taken matter!

Casc 8. Mr. Rice's child, case 197 of Dr. Rowley. I cotld obtain no other information, than that $M r$. Rice had been dead above a year, and the family removed, but could not learn where.

Cases $\mathbf{g}$ and 10. Elizabeth and William Keen; Dr. Rowley's 37 th and 38th cases; both of which he states thus: "Vaccinated May 10th, 1805 ; small-pox 29th of May."

I saw these two children, with their mother, in November 1805. Her relation was as follows:- She has three children, one of whom took the small-pox in the natural way, and had them very severely; and there was no doubt of the other two having caught the infection; but, by the advice of some friends, she had then vaccinated, hoping thercby to lessen the virulence of the small-pox. The result justified the procedure: the two vaccinated children passed through the small-pox so favourably, that their healths were scarcely impaired, and it was not easy to determine which of the two diseases predominated in the constitution; whereas the one that was not vaccinated, languished under a most distressing confluent small-pox, which left numerous indelible marks all over the face; besides a large and disgusting scar 
scar on the neck, just above the right clavicula; together with a general debility, which six months of time had hardly lessened.

Thus is seen, that these two cases, which Dr. Rowley has, by misrepresentation, endeavoured to warp to his own purposes, are most pointedly and strongly favourable to vaccination : how far it is so with the rest on his black list, will no duubt appear soon, from the labours of Dr. Thornton. I have much reason to believe they will, for the most part, be proved to be inisrepresentations of facts, in themselves neither for nor against vaccination : some few mistakes may have arisen from the inexperience of the early vaccinators,-this was to be expected by every rational person : many of the cases will be found highly favourable to vac, cination; but a considerable number of them contain their own refutation, which any one who reads with attention may discover. I am, sir,

Islington,

March 24, 1806.

Your humble servant,

John J. Hawkins.

XXXVII. On the Method of extracting Spirits from Potatoes. By M. Germain, Chemist to the Military Hospital at Hanau*.

$I_{\mathrm{T}}$ has been the practice, for a long time past, in Germany to distil spirits from potatoes. In the eastern part of Prussia, and in Lithuania, they employ an immense quantity of these vegetables in distillation. In these countries they are generally planted as the first crop in grounds which had been formerly untilled; and with proper care, and in good seasons, they produce abundantly. The residue, after distillation, is an excellent drink for cattle, particularly cows, whose milk is greatly increased by the use of it. When potatoe spirit is properly distilled, if not mixed with any foreign matter, and if the potatoes have not been heated too much, or burnt, during their preparation, it has a taste and

* From Annaies de Chimie, tome lvi. p. 207.

Vol. 24. No. 95. April 1806. 